\title{
Cardiovascular biomarkers in dogs with systemic inflammatory response syndrome
}

\author{
Kris Gommeren DVM, DECVIM, DECVECC ${ }^{1}$ (iD ｜｜Ｉsabelle Desmas DVM, DACVIM ${ }^{1}$ \\ Alexandra Garcia DVM ${ }^{1}$ | Cécile Clercx DVM, DECVIM, PhD ${ }^{1}$ | Kathleen Mc Entee DVM, \\ DECVIM, $\mathrm{PhD}^{2}$ | Anne-Christine Merveille DVM, $\mathrm{PhD}^{1}$ | Dominique Peeters DVM, \\ DECVIM, $\mathrm{PhD}^{1}$
}

${ }^{1}$ Department of Clinical Sciences, School of Veterinary Medicine, University of Liège, Liège, Belgium

${ }^{2}$ Faculty of Medicine, Laboratory of Physiology, Université Libre de Bruxelles, Brussels, Belgium

\section{Correspondence}

Kris Gommeren, Boulevard de Colonster 20, 4000 Liège, Belgium.

Email:krisjeg@hotmail.com

Presented in part at the 21st ECVIM-CA Congress, Seville, Spain, September 2011.

\begin{abstract}
Objective: To measure plasma N-terminal fragments of pro-B-type natriuretic peptides (NTproBNP) and cardiac troponin $\mathrm{T}$ (cTnT) concentration in hospitalized dogs and relate these markers to underlying conditions and evaluate their potential as prognostic markers in dogs with systemic inflammatory response syndrome (SIRS).

Design: Prospective, observational, clinical study.

Setting: Emergency department of a university teaching hospital.

Animals: Sixty-nine dogs with SIRS examined in the emergency department were prospectively studied. Patient age ranged from 5 months to 15 years, and weight ranged from 5.5 to $75 \mathrm{~kg}$.

Measurements and Main Results: Blood samples were obtained at presentation, during hospitalization until discharge or death, and at a "control" visit (T1m) at least 1 month after hospital discharge. NT-proBNP was assayed with a commercially available canine ELISA, while cTnT was measured with an automated immunoassay previously used in dogs. A correlation procedure, mixed procedure on a linear model, and a logistic procedure were performed. Forty-four patients survived, 19 of which had control visits. cTnT concentrations were significantly higher than TO and T1 $1 \mathrm{~m}$ at T12, T24, and T72. In 28 dogs, CTnT was detected during hospitalization, but CTnT was not detected in any dog at the control visits. Higher concentrations of cTnT were negatively associated with survival, irrespective of disease category. NT-proBNP concentrations were significantly higher than T0, T6, T12, and T1m at T24, T72, and T120, but were not associated with survival.

Conclusions: NT-proBNP and cTnT increased significantly in dogs with SIRS, regardless of the underlying disease process. Nonsurvivors displayed significantly higher cTnT concentrations during hospitalization.
\end{abstract}

KEYWORDS

brain natriuretic peptide, cardiac troponin, prognosis, SIRS

\section{1 | INTRODUCTION}

The systemic inflammatory response syndrome (SIRS) characterizes the systemic repercussions of inflammation. The list of underlying causes of SIRS is diverse, including sepsis, trauma, and sterile inflammatory conditions such as pancreatitis. ${ }^{1}$ The criteria for SIRS are based on defined changes in clinical (body temperature, heart rate, and respiratory rate) and hematologic (leukocyte counts, presence

Abbreviations: BNP, B-type natriuretic peptides; cTn, cardiac troponin; cTnl, cardiac troponin I; cTnT, cardiac troponin T; GDV, gastric dilation and volvulus; NT-proBNP, N-terminal fragment of pro-BNP; SIRS, systemic inflammatory response syndrome; $T^{\prime} X$ ', values obtained ' $X$ ' hours after presentation to the emergency department; $T 1 \mathrm{~m}$, 'control' visit at least 1 month after discharge.

(C) Veterinary Emergency and Critical Care Society 2019 
TAB LE 1 Clinical criteria for the diagnosis of SIRS (modified from Hauptman et al, ${ }^{2}$ with alterations in white blood cell count to adhere with the reference ranges of the clinical laboratory and temperature ranges as applied in clinics)

\begin{tabular}{lll} 
Parameter & Limit & Unit \\
Heart frequency & $>120$ & $\mathrm{bpm}$ \\
Respiratory rate & $<20$ & $\mathrm{rpm}$ \\
\hline Temperature & $<38$ or $>39$ & ${ }^{\circ} \mathrm{C}$ \\
\hline Leukocytosis/leukopenia & $>16,000$ or $<5,000$ & $/ \mu \mathrm{L}$ \\
\hline Left shift on blood smear & $>3$ & $\%$
\end{tabular}

of a left shift) variables (Table 1$)^{2}{ }^{2}$ The diagnosis of SIRS recognizes the presence of clinical signs compatible with systemic inflammation, but the criteria are overly sensitive and poorly specific. ${ }^{2}$ Cardiac dysfunction secondary to systemic inflammation has been reported in human medicine, and is also known as myocardial hibernation. ${ }^{3,4}$ Myocardial hibernation has been reported in critically ill human patients and studied in dogs with experimentally induced sepsis. ${ }^{4-7}$ It is characterized by increased end-diastolic and end-systolic ventricular volumes, ${ }^{8}$ and systolic ${ }^{3}$ and diastolic ${ }^{7}$ ventricular dysfunction. ${ }^{5}$ Whether myocardial hibernation serves as a protective mechanism of the body during systemic inflammation, or whether it has a negative impact on survival is unknown. ${ }^{5,9}$ Myocardial hibernation has been associated with increased blood concentrations of cardiac troponins (cTn) ${ }^{10,11}$ and natriuretic peptides ${ }^{12-14}$ in people with SIRS, and some studies have found concentrations of cTn and the $\mathrm{N}$-terminal portion of pro-brain natriuretic peptide (NT-proBNP) to be correlated with the degree of cardiac dysfunction and with plasma concentrations of inflammatory cytokines. ${ }^{9,15}$ Very little is known about myocardial hibernation in dogs with naturally occurring disease, although it is likely to occur. ${ }^{16,17}$ Recent data suggest that plasma concentrations of cardiac biomarkers are increased in dogs with SIRS. ${ }^{18-20}$ As these cardiac biomarkers may help in the diagnosis of cardiac dysfunction and the evaluation of prognosis in people with SIRS, 9,21,22 they may also be useful to evaluate dogs with SIRS.

Cardiac troponins $\mathrm{T}$ and I (cTnT and cTnl, respectively) are sensitive and specific for the detection of myocardial ischemic necrosis or reversible myocardial injury, and increased plasma cTnT and cTnl concentrations are associated with a poorer prognosis in critically ill people. ${ }^{23,24}$ Cardiac troponins have been studied in veterinary patients with cardiac disease, ${ }^{25}$ and are also increased and associated with poor prognosis in other syndromes such as gastric dilation and volvulus (GDV), ${ }^{25,26}$ trauma, ${ }^{25,27}$ infections, ${ }^{28-32}$ and SIRS. ${ }^{19,20,33}$

Brain natriuretic peptide (BNP) and NT-proBNP are quantitative markers of ventricular wall stress with high sensitivity and specificity for cardiac insult. ${ }^{12}$ Several studies have associated increases in plasma BNP and NT-proBNP in people with sepsis and SIRS with myocardial hibernation and poor prognosis., $92,13,15$ In veterinary medicine, increased plasma NT-proBNP concentrations have been demonstrated in dogs with babesiosis and in dogs with traumatic, neurological, and gastrointestinal diseases. ${ }^{32,34}$ We hypothesized that plasma cTnT and NT-proBNP would increase during hospitalization, vary in magnitude according to the underlying etiology, and serve as a prognostic marker in canine patients with SIRS.

\section{2 | MATERIALS AND METHODS}

All dogs that were examined in the emergency service of the University of Liège between January and August 2010 were considered for inclusion. Dogs were enrolled if SIRS was diagnosed based on the suspicion of an underlying disease process known to trigger the systemic inflammatory response and the finding of 2 or more abnormalities of the following clinical (temperature, heart rate, and respiratory rate) and basic laboratory parameters (leukocyte counts). ${ }^{2}$ The cutoff values for white blood cell counts were modified from the original paper to adhere with the reference ranges of our own clinical laboratory (Table 1 ) and the limits of normal rectal temperature were between 38 and $39^{\circ} \mathrm{C}$. Approval for this project was obtained from the ethics committee (approval number 1709), and informed consent was obtained from the owners of each dog. Exclusion criteria included a weight $<5 \mathrm{~kg}$ and animals that were considered too unstable to sustain any additional or unnecessary stress. Patients were grouped into 7 different disease categories: patients with neoplastic disease $(N)$, infectious disease (I), GDV, gastrointestinal disease (GI), trauma (T), renal disease (R), and miscellaneous causes (M). Since concentrations of plasma NT-proBNP can be influenced by renal function, patients with renal insufficiency (defined as azotemia or oligo-anuria that was unresponsive to fluid therapy) were excluded from NT-proBNP analysis. ${ }^{35}$ Although plasma cTn concentrations also can be influenced by renal function, the severity of renal failure does not correlate with cTn concentrations, ${ }^{36,37}$ and cTn analysis remains useful in identifying myocardial injury in people with renal disease. ${ }^{38-40}$ Similarly, plasma cTn concentrations can identify human patients with poorer prognosis despite concurrent renal failure or requirement for hemodialysis. ${ }^{38,41-46}$ Based on these findings, we did not exclude patients with renal impairment from plasma cTnT analysis.

Baseline plasma concentrations of cTnT and NT-proBNP were assessed on blood sampled prior to starting any treatment (TO), and on samples obtained after 6 (T6), 12 (T12), 24 hours (T24), and every other day thereafter (T72, T120, etc.) until hospital discharge or death. Short-term survival was defined as patient discharge from the hospital, while long-term survival was defined as the patient being alive 1 month after discharge from the hospital. All long-term survivors were invited to a recheck "control" examination occurring between 1 month and 1 year after hospital discharge, where blood was again collected for analysis. All blood samples were divided into EDTA ( $4 \mathrm{~mL})$ and serum $(2 \mathrm{~mL}$ ) tubes that were centrifuged and separated within 15 minutes, with the serum or plasma stored at $-80^{\circ} \mathrm{C}$ until analysis.

A commercial electrochemiluminescence kit,* with a lower limit of detection of $0.010 \mathrm{ng} / \mathrm{mL}$, a limit of linearity of $25.00 \mathrm{ng} / \mathrm{mL}$, and a coefficient of variation less than $5 \%$ for values above $0.06 \mathrm{ng} / \mathrm{mL}$, was used to measure serum cTnT. ${ }^{47}$ The kit detected 2 epitopes of the central part of human cTnT (125-131 and 135-147), which are highly conserved in canine cTnT (one substitution in the first epitope and 
$100 \%$ homology in the second), and has previously been used in veterinary research. ${ }^{47,48}$ Reported values in healthy dogs are less than $0.010 \mathrm{ng} / \mathrm{mL} .^{27,49}$

A commercially available sandwich enzyme immune assay ${ }^{\dagger}$ with an upper limit of detection of $3,000 \mathrm{pmol} / \mathrm{L}$ was used to measure NTproBNP. Patient plasma and an immunoaffinity purified sheep detection antibody conjugated to horseradish peroxidase were incubated for 5 hours at $20^{\circ} \mathrm{C}$ in microtiter plates with capture antibody (antiNT-proBNP) bound to the wells. The wells were then washed and tetramethylbenzidine was added and incubated for 40 minutes, after which a stop solution was added and bound NT-proBNP was quantified by an ELISA plate reader. All plates were run with calibration and control solutions, yet for financial reasons only the first plate was run in duplicate.

\section{1 | Statistical methods}

Statistical analysis was performed using a commercially available statistics program. A Shapiro-Wilk and Kolmogorov-Smirnov test (univariate procedure) and normality QQplots were performed on the raw data and on logarithmic-transformed data. For both cTnT and NTproBNP, the logarithmically transformed data were used for analysis after identification of a nearly normal distribution of the residues on the QQplots. A mixed procedure on a generalized linear model was used to assess the relationship of measured clinical parameters to cardiac biomarkers. As the data were taken repeatedly over time on the same animals, there is a possible correlation between successive data. This correlation structure is reflected in the linear mixed model used (MIXED procedure, repeated by time). Correlation between different biomarkers was tested (CORR procedure). A logistic analysis (LOGISTIC procedure) was performed in order to evaluate the association of plasma cardiac biomarker concentrations with survival to discharge. Only dogs that survived, died of natural causes, or were euthanized for prognostic reasons were included in the assessment of prognostic value of the evaluated parameters. Statistical significance was set at a $P$-value $<0.05$

\section{3 | RESULTS}

Fifty-eight purebred and 11 mixed-breed dogs were included in the study. The most commonly represented breeds were Bernese Mountain Dog $(n=8)$, German Shepherd Dog $(n=6)$, Great Dane $(n=4)$, Jack Russell Terrier $(n=4)$, and Belgian Shepherd Dog $(n=3)$. There were 38 male (29 intact and 9 neutered) and 31 female (17 intact and 14 neutered) dogs with a median age of 6.5 years (ranging between 7 months and 15.2 years) and with a median weight of $30.3 \mathrm{~kg}$ (ranging from 5.5 to $75 \mathrm{~kg})$. Patients were categorized by disease $(\mathrm{N}=13$; I = $12 ; \mathrm{GDV}=11 ; \mathrm{GI}=5 ; \mathrm{T}=6 ; \mathrm{R}=3$; and $\mathrm{M}=19$ ). Outcome and followup of our studied population has been represented in a flow diagram (Figure 1). Forty-four patients were discharged, 8 died during hospitalization, and 17 dogs were euthanized ( 8 for prognostic, 7 for financial, and 2 for unspecified reasons). Thirty-four patients were still alive more than 1 month after discharge and 19 were presented for a control

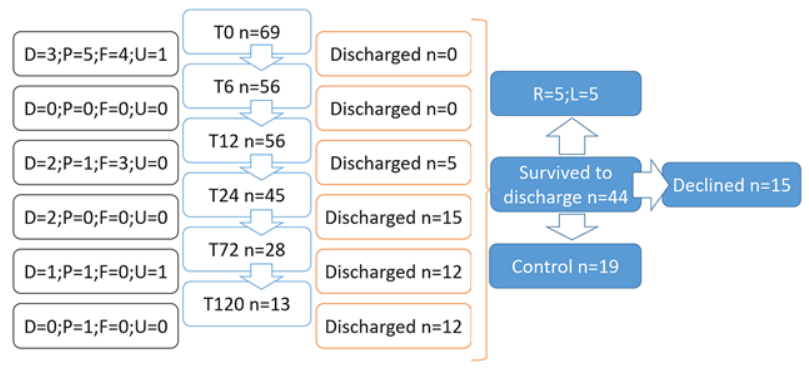

FIGURE 1 Flow diagram of the disposition of dogs with SIRS throughout the study. D, deceased; P, euthanized for prognostic reasons; $F$, euthanized for financial reasons; $U$, euthanized for unclear reasons; $\mathrm{R}$, died more than a month after discharge yet before a control visit was performed; L, lost to follow-up

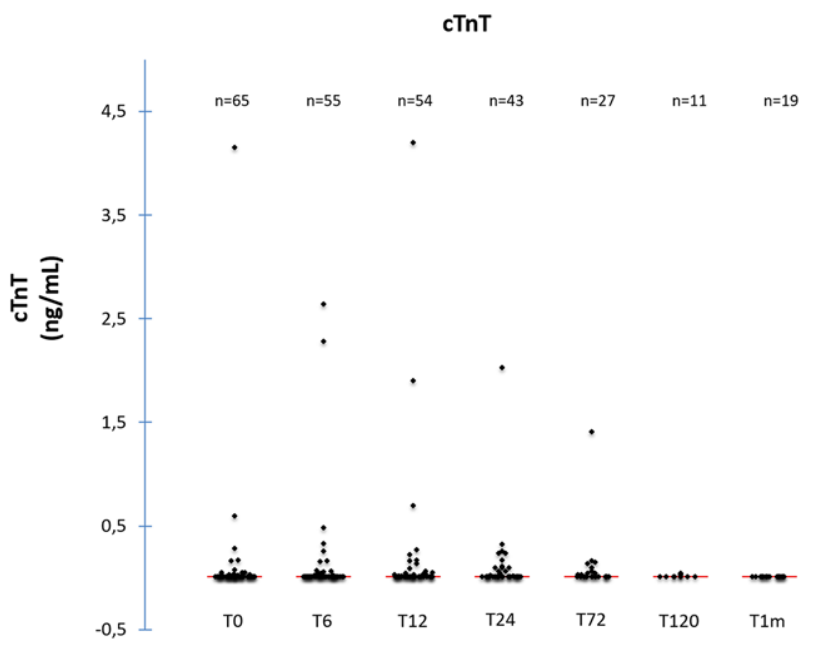

FIGURE 2 Scatter plots of serum concentrations of cTnT at different time points in hospitalized dogs with SIRS. The red line indicates the median value

visit. Of the dogs that did not have a follow-up visit, 5 died from related causes before the scheduled visit (including continued gastrointestinal illness in 2 dogs, aspiration pneumonia secondary to a megaesophagus, worsening hepatocutaneous syndrome, and tumor recurrence with secondary hemoabdomen in 1 dog each), 5 owners declined, and 5 dogs were lost to follow-up.

\section{1 | Biomarker analysis}

Plasma concentrations of cTnT and NT-proBNP both changed significantly over time $(P<0.0001)$. Concentrations of cTnT and NT-proBNP at each timepoint are displayed in Figures 2 and 3, respectively. Twenty-eight of 69 dogs had at least 1 time point during hospitalization at which cTnT was detectable, while none of the dogs had measurable cTnT concentrations at the control visit. Plasma cTnT concentration was significantly higher at T12, T24, and T72 compared to concentrations at presentation and at the control visit (Table 2). Plasma NT-proBNP concentrations were measurable in all dogs at all timepoints. Plasma NT-proBNP concentrations were significantly higher at T24, T72, and T120 compared to T0, T6, T12, and T1m. Median concentrations did not differ significantly between T24 


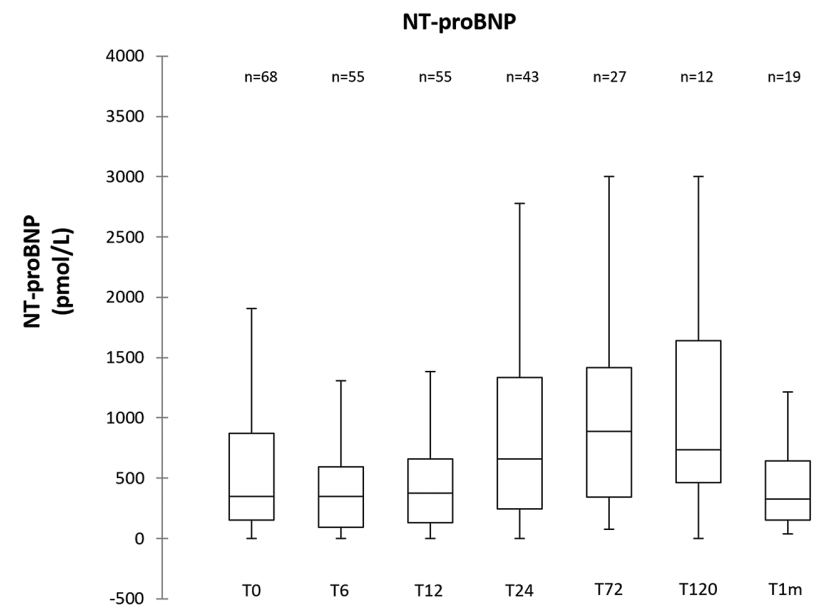

FIGURE 3 Plasma concentrations of NT-proBNP at different time points in dogs with SIRS. The central line of the box plot indicates the median value, the upper and lower line of the box plot illustrate the range of the $25 \%$ and $75 \%$ of the values, and the outer lines at the end of the vertical lines indicate the $95 \%$ and $5 \%$ range of the recorded values

(661.39 [60.77-3,000] pmol/L), T72 (888.81 [76.58-3,000] pmol/L), and T120 (737.14 [0-3,000] pmol/L; Table 3).

\subsection{Correlation of biomarkers with underlying disease, prognosis, and each other}

Statistical analysis did not identify any influence of the underlying disease category on cTnT and NT-proBNP concentrations $(P=0.1618$ and 0.084 , respectively). Regardless of the specific timepoint, detectable

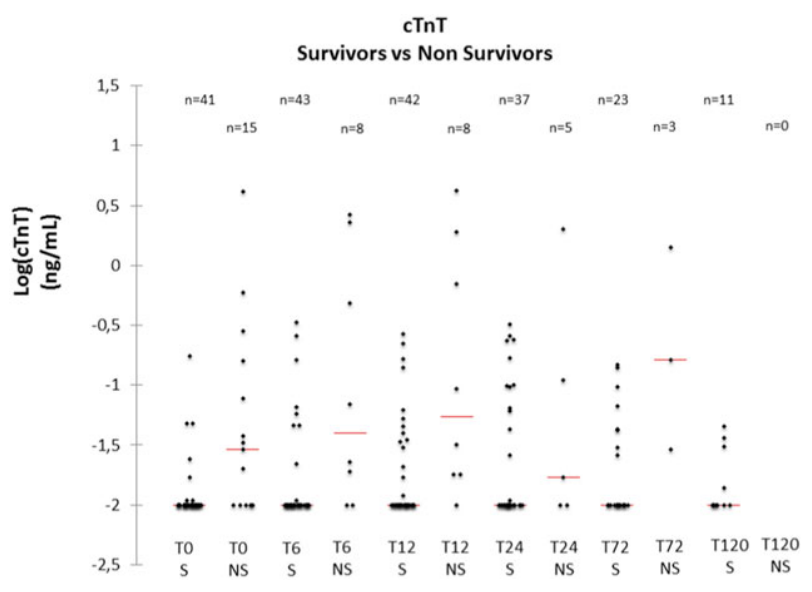

FIGURE 4 Scatter plot of serum cardiac troponin T (cTnT) concentrations in survivors and nonsurvivors at different timepoints; S, survivor; NS, nonsurvivor (natural death or euthanized for prognostic reasons). The red line indicates the median value

cTnT concentrations $(P=0.0106)$ were associated with poor prognosis (Figure 4). In contrast, NT-proBNP concentrations were not correlated with survival to discharge $(P=0.5091$, Figure 5$)$. Finally, concentrations of cTnT and NT-proBNP were significantly and mildly correlated $(P<0.001$, with $R=0.2913)$.

\section{4 | DISCUSSION}

This study demonstrated changes in cardiac biomarkers during hospitalization in a population of canine patients with SIRS. Troponin

TA B LE 2 P-values for CTnT concentrations between different time points in all canine SIRS patients

\begin{tabular}{|c|c|c|c|c|c|c|c|}
\hline & TO & T6 & $\mathrm{T} 12$ & T24 & T72 & T120 & $\mathrm{T} 1 \mathrm{~m}$ \\
\hline TO & 1 & 0.0501 & $0.0002 *$ & $0.0004 *$ & $0.0231 *$ & 0.8514 & 0.0866 \\
\hline T6 & 0.0501 & 1 & 0.0677 & 0.0748 & 0.4441 & 0.368 & $0.0022 *$ \\
\hline T24 & $0.0004 *$ & 0.0748 & 0.9257 & 1 & 0.4769 & 0.0536 & $<0.0001 *$ \\
\hline T72 & $0.0231 *$ & 0.4441 & 0.514 & 0.4769 & 1 & 0.174 & $0.001 *$ \\
\hline T1m & 0.0866 & $0.0022 *$ & $<0.0001 *$ & $<0.0001 *$ & $0.001 *$ & 0.1634 & 1 \\
\hline
\end{tabular}

Significant differences are indicated in bold with an asterisk $(*)$.

TAB LE $3 \quad P$-values for NT-proBNP concentrations between different time points in all canine SIRS patients

\begin{tabular}{|llllllll} 
& T0 & T6 & T12 & T24 & T72 & T120 & T1m \\
\hline T0 & 1 & 0.2712 & 0.2125 & $0.008 *$ & $0.0004 *$ & $0.005 *$ & 0.9357 \\
\hline T6 & 0.2712 & 1 & 0.8849 & $0.0003 *$ & $<0.0001 *$ & $0.0007 *$ & 0.4015 \\
\hline T12 & 0.2125 & 0.8849 & 1 & $0.0002 *$ & $<0.0001 *$ & $0.0005 *$ & 0.3475 \\
\hline T24 & $0.008 *$ & $0.0003 *$ & $0.0002 *$ & 1 & 0.2061 & 0.2321 & 0.0626 \\
\hline T72 & $0.0004 *$ & $<0.0001 *$ & $<0.0001 *$ & 0.2061 & 1 & 0.7928 & $0.0063 *$ \\
\hline T120 & $0.005 *$ & $0.0007 *$ & $0.0005 *$ & 0.2321 & 0.7928 & 1 & $0.0155 *$ \\
\hline T1m & 0.9357 & 0.4015 & 0.3475 & 0.0626 & $0.0063 *$ & $0.0155 *$ & 1 \\
\hline
\end{tabular}

Significant differences are indicated in bold with an asterisk $(*)$. 


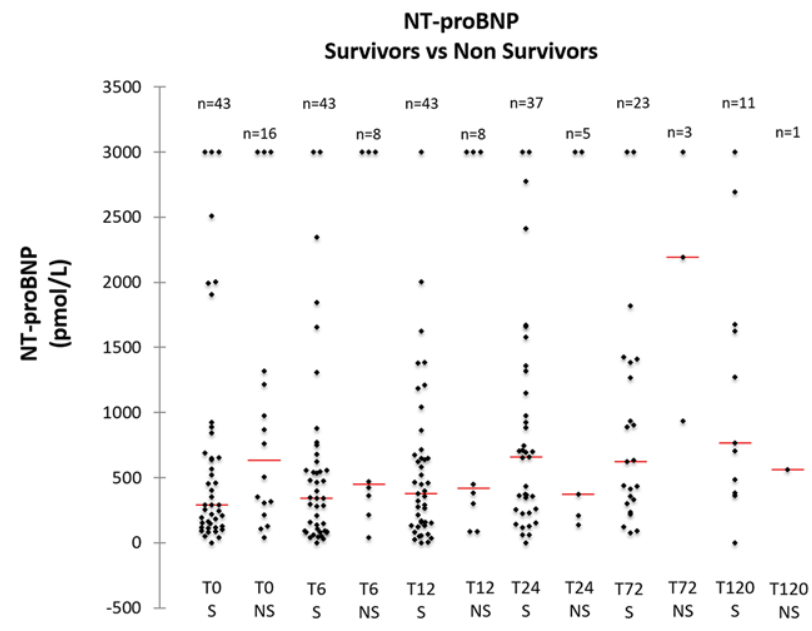

FIGURE 5 Plasma concentrations of NT-proBNP in survivors and non survivors at different time points; S, survivor; NS, nonsurvivor (natural death or euthanized for prognostic reasons). The red line indicates the median value

concentrations rise within 8 hours after an initial insult, and remain increased for over 50 hours in people and dogs. ${ }^{10,50-52}$ cTnT values in the cohort of dogs in this report were detectable in 28 dogs during hospitalization, with concentrations significantly higher at T12, T24, and T72, compared to concentrations at presentation and at the control visit. Although the clinical nature of this study on dogs suffering from different diseases does not allow us to identify the exact timing of the original insult in the majority of dogs, the timing of the changes in cTnT concentrations appear to agree with the rapid rise and sustained increase described in other reports. In contrast, at the control visit, all dogs had undetectable cTnT concentration $(<0.01 \mathrm{ng} / \mathrm{mL})$. A study in dogs with GDV described similar findings, with no significant changes in cTnT concentrations immediately after surgery but increased concentrations on the 2 days after presentation. ${ }^{25,26}$ A study evaluating cTnl concentrations in dogs with SIRS identified a higher prevalence of increased cTnl concentrations at presentation (35/60 dogs) and during hospitalization, but also did not find significant variations from day to day. ${ }^{19}$ A study comparing cTnT and cTnl in dogs with SIRS admitted to an ICU found a higher prevalence of increased cTnl concentrations. This difference in detection rate can be explained by the lower sensitivity of cTnT compared to cTnl to detect myocardial lesions, or by the timing of sampling compared to the start of the disease process (as admission to the ICU likely takes place later than admission to an emergency department). ${ }^{20}$ The use of a cTnl assay in the present study may have resulted in the detection of increased concentrations in a larger proportion of SIRS patients, but cTnT was chosen for financial reasons. ${ }^{26,28}$

NT-proBNP changed significantly over time, with concentrations at T24, T72, and T120 significantly higher than concentrations at T0, T6, T12, and the control visit. Most of the research performed in veterinary medicine on NT-proBNP has focused on cardiac disease. ${ }^{53-55}$ A recent study evaluating BNP in dogs with noncardiac disease (eg, neurological and gastrointestinal disease) demonstrated a moderate increase of natriuretic peptides in these patients. ${ }^{34}$ BNP and
NT-proBNP concentrations cannot be directly compared. However, as SIRS has a high potential to induce cardiac effects in human medicine, the more markedly increased NT-proBNP concentrations in our study focused on dogs with SIRS compared to this previous study therefore do not seem surprising. ${ }^{35}$ Finding higher concentrations at T24, T72, and T120 is in agreement with studies on SIRS and sepsis in human patients. The time point with the highest plasma NT-proBNP measurement varied across studies in people, from the day of admission to day 2 and day 5 after admission, ${ }^{22,56}$ which is probably due to the difficulty in determining the time of onset of the disease. Nevertheless, peak concentrations are likely to be found more than 2 days after hospitalization in people.9,15,21 Unfortunately, the clinical setting of this study prevented determination of the exact time the insult triggering SIRS occurred for the majority of dogs. The kinetics observed in this cohort do confirm that NTproBNP is expected to rise during the first days of hospitalization in dogs with SIRS. Increased concentrations of NT-proBNP when screening for occult cardiac disease should therefore be interpreted carefully in patients that also have SIRS. Whether increased cTnT and NTproBNP concentrations are indicative of myocardial hibernation in dogs with SIRS cannot be concluded from this paper but merits further investigation.

Detectable cTnT concentrations during hospitalization were associated with poor short-term prognosis. Cardiac troponin T and I are well-accepted prognostic biomarkers in human intensive care units. ${ }^{10,11,23,57}$ In veterinary medicine, increased concentrations of cTns have been observed in infectious disease patients, trauma patients, GDV patients, and patients suffering from systemic diseases $25,26,28-30,58-62$ and are correlated with poor prognosis in some of these reports. ${ }^{25,26,28,30}$ Reports that evaluate plasma concentrations of $\mathrm{cTnl}$ and $\mathrm{cTnT}$ in dogs with SIRS have confirmed their prognostic value, identifying differences in concentration between survivors and nonsurvivors. ${ }^{19,20,33}$ In one report, additional sampling to measure cTnl concentrations on day 2 or 3 (or evaluation of concentration changes) did not add value to the prognostic information. ${ }^{19}$ Although the incidence of increased $\mathrm{cTnl}$ concentrations has been reported to be higher than for $\mathrm{cTnT}, \mathrm{cTnl}$ and $\mathrm{cTnT}$ carried similar prognostic information. ${ }^{20,33}$ cTnT concentrations have been established as interesting markers to predict prognosis of canine SIRS patients, but cutoff limits remain to be determined. ${ }^{33}$ As cTnT (and cTnl) can remain increased up to 7 or 10 days after an insult, the kinetics of cTnT are difficult to evaluate, and they may be less useful for evaluation of disease progression. ${ }^{63,64}$

In the present study, NT-proBNP concentrations were not significantly correlated with prognosis. Previous studies in dogs tended to evaluate natriuretic peptides at presentation, while higher concentrations should be expected later during hospitalization. This delay in the rise of NT-proBNP limits its use as a prognostic marker in a clinical veterinary emergency care setting. At later timepoints, the group size in this study rapidly decreased, which may have impacted the likelihood to identify significant differences between survivors and nonsurvivors. A recently published meta-analysis in human patients with sepsis concluded that NT-proBNP concentrations are significantly associated with risk of mortality. ${ }^{22}$ This meta-analysis also 
concluded that increased NT-proBNP concentrations in the presence of SIRS or sepsis do not indicate cardiac dysfunction, but normal NT-proBNP concentrations rule out the need for further cardiac investigation. ${ }^{22}$ The lack of a significant difference in NT-proBNP concentration between survivors and nonsurvivors in this study should be confirmed in a larger cohort of patients with SIRS. The observed increased NT-proBNP concentrations in this study may be explained by myocardial hibernation, but also may have been increased due to increased cardiac wall stress after volume resuscitation, ${ }^{65}$ the presence of lung injury, acute respiratory distress syndrome, or thromboembolism. ${ }^{6}$

There are several limitations to the present study. Dogs that were considered too unstable by the attending clinician were removed from the study, and therefore, more severely ill patients were less likely to enter the study. Consequently, findings might have been different if all dogs were included, regardless of their clinical status. Because sampling times were standardized with relation to the timing of presentation to the emergency department, disease may have been present for variable amounts of time prior to enrollment, and this may have altered the kinetics of the studied biomarkers. In addition, a large proportion of our patients were euthanized at the owner's request, rather than based on specific study endpoints, which may have impacted our analysis of prognosis. In order to avoid any effect of financial considerations on the prognostic evaluation, all dogs that were euthanized for financial or unspecified reasons were removed from the prognosis analysis. In the present study, 44 patients survived to discharge (64\%), which is similar to, ${ }^{66}$ or better than ${ }^{67}$ previous studies on clinical canine SIRS patients.

Samples with NT-proBNP concentrations above the upper limit of the assay $(3,000 \mathrm{pmol} / \mathrm{L})$ were not diluted to measure the exact concentration because of financial restrictions. Therefore, NT-proBNP concentration was underestimated in some samples. This did not prohibit the finding of significant changes, but may have underestimated the degree of those changes in NT-proBNP concentration. Similarly, a cTnT assay rather than a cTnl assay was used due to financial restrictions. The use of a cTnl assay may have resulted in a higher detection rate of increased troponin concentrations.

Our study demonstrates that cardiac biomarkers are often increased in dogs with SIRS presenting to the emergency department. Additionally, this study confirms that the cTnT concentration was associated with survival in dogs with SIRS. Studies investigating the correlation of cardiac biomarkers with echocardiographic findings and inflammatory cytokines in canine patients with SIRS are warranted.

\section{CONFLICT OF INTEREST}

The authors declare no conflict of interest.

\section{ENDNOTES}

* Modular Analytics E, Roche, Basel, Switzerland
+ VetSign Canine Cardioscreen Test Kit, IDEXX Laboratories, Westbrook,
ME

* SAS; Statistical Analysis Software, Cary, NC

\section{ORCID}

Kris Gommeren DVM, DECVIM, DECVECC

https://orcid.org/0000-0003-1647-1002

\section{REFERENCES}

1. de Laforcade AM. Systemic inflammatory response syndrome. In: Silverstein DC, Hopper K, eds. Small Animal Critical Care Medicine. 2nd ed. St. Louis, MO: Saunders Elsevier; 2015:31-34.

2. Hauptman JG, Walshaw R, Olivier NB. Evaluation of the sensitivity and specificity of diagnostic criteria for sepsis in dogs. Vet Surg. 1997;26:393-397.

3. Parker M, Shelhamer J, Bacharach S, et al. Profound but reversible myocardial depression in patients with septic shock. Ann Intern Med. 1984;100:483-490.

4. Werdan $\mathrm{K}$, Schmidt $\mathrm{H}$, Ebelt $\mathrm{H}$, et al. Impaired regulation of cardiac function in sepsis, SIRS, and MODS. Can J Physiol Pharmacol. 2009;87:266-274.

5. Levy R, Piel D, Acton P, et al. Evidence of myocardial hibernation in the septic heart. Crit Care Med. 2005;33:2752-2756.

6. Maeder M, Fehr T, Rickli H, et al. Sepsis-associated myocardial dysfunction: diagnostic and prognostic impact of cardiac troponins and natriuretic peptides. Chest. 2006;129:1349-1366.

7. Natanson C, Fink M, Ballantyne H, et al. Gram-negative bacteremia produces both severe systolic and diastolic cardiac dysfunction in a canine model that simulates human septic shock. J Clin Invest. 1986;78:259-270.

8. Marik P, Varon J. Sepsis: state of the art. Dis Mon. 2001;47:465-532

9. Charpentier J, Luyt CE, Fulla Y, et al. Brain natriuretic peptide: a marker of myocardial dysfunction and prognosis during severe sepsis. Crit Care Med. 2004;32:660-665.

10. Babuin L, Vasile V, Rio Perez J, et al. Elevated cardiac troponin is an independent risk factor for short- and long-term mortality in medical intensive care unit patients. Crit Care Med. 2008;36:759-765.

11. Ammann $P$, Maggiorini $M$, Bertel $O$, et al. Troponin as a risk factor for mortality in critically ill patients without acute coronary syndromes. $J$ Am Coll Cardiol. 2003;41:2004-2009.

12. Chen $Y$, Li C. Prognostic significance of brain natriuretic peptide obtained in the ED in patients with SIRS or sepsis. Am J Emerg Med. 2009;27:701-706.

13. Meyer B, Huelsmann M, Wexberg P, et al. N-terminal pro-B-type natriuretic peptide is an independent predictor of outcome in an unselected cohort of critically ill patients. Crit Care Med. 2007;35:2268-2273.

14. Rudiger A, Fischler $M$, Harpes $P$, et al. In critically ill patients, Btype natriuretic peptide (BNP) and $\mathrm{N}$-terminal pro-BNP levels correlate with C-reactive protein values and leukocyte counts. Int J Cardiol. 2008;126:28-31

15. Witthaut R, Busch C, Fraunberger $P$, et al. Plasma atrial natriuretic peptide and brain natriuretic peptide are increased in septic shock: impact of interleukin-6 and sepsis-associated left ventricular dysfunction. Intensive Care Med. 2003;29:1696-1702.

16. Dickinson A, Rozanski E, Rush J. Reversible myocardial depression associated with sepsis in a dog. J Vet Intern Med. 2007;21:1117-1120.

17. Nelson $O$, Thompson P. Cardiovascular dysfunction in dogs associated with critical illnesses. J Am Anim Hosp Assoc. 2006;42:344-349.

18. Langhorn R, Persson F, Åblad B, et al. Myocardial injury in dogs with snake envenomation and its relation to systemic inflammation. J Vet Emerg Crit Care. 2014;24:174-181. 
19. Hamacher L, Dorfelt R, Muller M, et al. Serum cardiac troponin I concentrations in dogs with systemic inflammatory response syndrome. $J$ Vet Intern Med. 2015;29:164-170.

20. Langhorn R, Oyama MA, King LG, et al. Prognostic importance of myocardial injury in critically ill dogs with systemic inflammation. J Vet Intern Med. 2013;27:895-903.

21. Roch A, Allardet-Servent J, Michelet $\mathrm{P}$, et al. NH2 terminal probrain natriuretic peptide plasma level as an early marker of prognosis and cardiac dysfunction in septic shock patients. Crit Care Med. 2005;33:1001-1007.

22. Wang $\mathrm{F}, \mathrm{Wu} \mathrm{Y}$, Tang $\mathrm{L}$, et al. Brain natriuretic peptide for prediction of mortality in patients with sepsis: a systematic review and metaanalysis. Crit Care. 2012;16:R74.

23. Spies C, Haude V, Fitzner R, et al. Serum cardiac troponin T as a prognostic marker in early sepsis. Chest. 1998;113:1055-1063.

24. Turner A, Tsamitros M, Bellomo R. Myocardial cell injury in septic shock. Crit Care Med. 1999;27:1775-1780.

25. Burgener IA, Kovacevic A, Mauldin GN, et al. Cardiac troponins as indicators of acute myocardial damage in dogs. $J$ Vet Intern Med. 2006;20:277-283.

26. Schober KE, Cornand C, Kirbach B, et al. Serum cardiac troponin I and cardiac troponin $\mathrm{T}$ concentrations in dogs with gastric dilatationvolvulus. J Am Vet Med Assoc. 2002;221:381-388.

27. Schober KE, Kirbach B, Oechtering G. Noninvasive assessment of myocardial cell injury in dogs with suspected cardiac contusion. J Vet Cardiol. 1999;1:17-25.

28. Lobetti R, Dvir E, Pearson J. Cardiac troponins in canine babesiosis. J Vet Intern Med. 2002;16:63-68.

29. Diniz PP, de Morais HS, Breitschwerdt EB, et al. Serum cardiac troponin I concentration in dogs with ehrlichiosis. J Vet Intern Med. 2008;22:1136-1143.

30. Mastrorilli C, Dondi F, Agnoli C, et al. Clinicopathologic features and outcome predictors of Leptospira interrogans Australis serogroup infection in dogs: a retrospective study of 20 cases (2001-2004). J Vet Intern Med. 2007;21:3-10.

31. Silvestrini P, Piviani M, Alberola J, et al. Serum cardiac troponin I concentrations in dogs with leishmaniasis: correlation with age and clinicopathologic abnormalities. Vet Clin Pathol. 2012;41:568-574.

32. Lobetti R, Kirberger R, Keller N, et al. NT-ProBNP and cardiac troponin I in virulent canine babesiosis. Vet Parasitol. 2012;190:333339.

33. Langhorn R, Thawley V, Oyama MA, et al. Prediction of long-term outcome by measurement of serum concentration of cardiac troponins in critically ill dogs with systemic inflammation. J Vet Intern Med. 2014;28:1492-1497.

34. Lee JA, Herndon WE, Rishniw M. The effect of noncardiac disease on plasma brain natriuretic peptide concentration in dogs. J Vet Emerg Crit Care. 2011;21:5-12.

35. Boswood A, Dukes-McEwan J, Loureiro J, et al. The diagnostic accuracy of different natriuretic peptides in the investigation of canine cardiac disease. J Small Anim Pract. 2008;49:26-32.

36. De Zoysa JR. Cardiac troponins and renal disease. Nephrology (Carlton). 2004;9:83-88.

37. Lamb EJ, Webb MC, Abbas NA. The significance of serum troponin $\mathrm{T}$ in patients with kidney disease: a review of the literature. Ann Clin Biochem. 2004;41:1-9.

38. Martin GS, Becker BN, Schulman G. Cardiac troponin-I accurately predicts myocardial injury in renal failure. Nephrol Dial Transplant. 1998;13:1709-1712.
39. McCullough PA, Nowak RM, Foreback C, et al. Performance of multiple cardiac biomarkers measured in the emergency department in patients with chronic kidney disease and chest pain. Acad Emerg Med. 2002;9:1389-1396.

40. McLaurin MD, Apple FS, Falahati A, et al. Cardiac troponin I and creatine kinase-MB mass to rule out myocardial injury in hospitalized patients with renal insufficiency. Am J Cardiol. 1998;82:973-975.

41. Rahman A, Broadley SA. Review article: elevated troponin: diagnostic gold or fool's gold? Emerg Med Australas. 2014;26:125-130.

42. Apple FS, Murakami MM, Pearce LA, et al. Predictive value of cardiac troponin I and T for subsequent death in end-stage renal disease. Circulation. 2002;106:2941-2945.

43. Ooi DS, Zimmerman D, Graham J, et al. Cardiac troponin T predicts long-term outcomes in hemodialysis patients. Clin Chem. 2001;47:412-417.

44. Apple FS, Sharkey SW, Hoeft $P$, et al. Prognostic value of serum cardiac troponin I and T in chronic dialysis patients: a 1-year outcomes analysis. Am J Kidney Dis. 1997;29:399-403.

45. Antman EM, Tanasijevic MJ, Thompson B, et al. Cardiac-specific troponin I levels to predict the risk of mortality in patients with acute coronary syndromes. N Engl J Med. 1996;335:1342-1349.

46. Stolear JC, Georges B, Shita A, et al. The predictive value of cardiac troponin T measurements in subjects on regular haemodialysis. Nephrol Dial Transplant. 1999;14:1961-1967.

47. Osathanon R, Moonarmart W, Suksantilap N, et al. Evaluation of hematology profiles and measurement of serum cardiac troponin level in canine monocytic ehrlichiosis. Thai J Vet Med. 2013;43:405-409.

48. Giannitsis E, Kurz K, Hallermayer K, et al. Analytical validation of a high-sensitivity cardiac troponin T assay. Clin Chem. 2010;56:254261.

49. DeFrancesco TC, Atkins CE, Keene BW, et al. Prospective clinical evaluation of serum cardiac troponin $\mathrm{T}$ in dogs admitted to a veterinary teaching hospital. J Vet Intern Med. 2002;16:553-557.

50. Fonfara S, Loureiro J, Swift S, et al. Cardiac troponin I as a marker for severity and prognosis of cardiac disease in dogs. Vet J. 184:334-339.

51. Feng $X$, Taggart $P$, Hall $L$, et al. Limited additional release of cardiac troponin I and T in isoproterenol-treated beagle dogs with cardiac injury. Clin Chem. 2005;51:1305-1307.

52. Wu A. Increased troponin in patients with sepsis and septic shock: myocardial necrosis or reversible myocardial depression? Intensive Care Med. 2001;27:959-961.

53. Oyama MA, Sisson DD, Solter PF. Prospective screening for occult cardiomyopathy in dogs by measurement of plasma atrial natriuretic peptide, B-type natriuretic peptide, and cardiac troponin-I concentrations. Am J Vet Res. 2007;68:42-47.

54. Hori Y, Tsubaki M, Katou A, et al. Evaluation of NT-pro BNP and CTANP as markers of concentric hypertrophy in dogs with a model of compensated aortic stenosis. J Vet Intern Med. 2008;22:1118-1123.

55. Noszczyk-Nowak A. NT-pro-BNP and troponin I as predictors of mortality in dogs with heart failure. Pol J Vet Sci. 2011;14:551-556.

56. Fromm RJ, Varon J. NH2 terminal pro-brain natriuretic peptide in cardiovascular dysfunction and septic shock. Crit Care Med. 2005;33:1156-1157.

57. Wu TT, Yuan A, Chen CY, et al. Cardiac troponin I levels are a risk factor for mortality and multiple organ failure in noncardiac critically ill patients and have an additive effect to the APACHE II score in outcome prediction. Shock. 2004;22:95-101.

58. Prosek R, Sisson DD, Oyama MA, et al. Distinguishing cardiac and noncardiac dyspnea in 48 dogs using plasma atrial natriuretic factor, 
B-type natriuretic factor, endothelin, and cardiac troponin-I. J Vet Intern Med. 2007;21:238-242.

59. Hagman R, Lagerstedt AS, Fransson BA, et al. Cardiac troponin I levels in canine pyometra. Acta Vet Scand. 2007;49:6.

60. Porciello F, Rishniw M, Herndon WE, et al. Cardiac troponin I is elevated in dogs and cats with azotaemia renal failure and in dogs with non-cardiac systemic disease. Aust Vet J. 2008;86:390-394.

61. Pelander L, Hagman R, Häggström J. Concentrations of cardiac troponin I before and after ovariohysterectomy in 46 female dogs with pyometra. Acta Vet Scand. 2008;50:35.

62. Barr S, Warner K, Kornreic B, et al. A cysteine protease inhibitor protects dogs from cardiac damage during infection by Trypanosoma cruzi. Antimicrob Agents Chemother. 2005;49:5160-5161.

63. O'Brien PJ, Dameron GW, Beck ML, et al. Cardiac troponin T is a sensitive, specific biomarker of cardiac injury in laboratory animals. Lab Anim Sci. 1997;47:486-495.

64. Katus HA, Remppis A, Scheffold T, et al. Intracellular compartmentation of cardiac troponin $\mathrm{T}$ and its release kinetics in patients with reperfused and nonreperfused myocardial infarction. Am J Cardiol. 1991;67:1360-1367.

65. Phua J, Lim TK, Lee KH. B-type natriuretic peptide: issues for the intensivist and pulmonologist. Crit Care Med. 2005;33:2094-2013.

66. Rau S, Kohn B, Richter C, et al. Plasma interleukin-6 response is predictive for severity and mortality in canine systemic inflammatory response syndrome and sepsis. Vet Clin Pathol. 2007;36:253-260.

67. $\mathrm{Yu} \mathrm{DH}, \mathrm{Nho} \mathrm{DH}$, Song RH, et al. High-mobility group box 1 as a surrogate prognostic marker in dogs with systemic inflammatory response syndrome. J Vet Emerg Crit Care. 2010;20:298-302.

How to cite this article: Gommeren K, Desmas I, Garcia A, et al. Cardiovascular biomarkers in dogs with systemic inflammatory response syndrome. J Vet Emerg Crit Care. 2019;29:256-263. https://doi.org/10.1111/vec.12833 